\title{
Round table discussion of session A: modelling convection and radiative transfer
}

\author{
F. Kupka ${ }^{1}$ \\ ${ }^{1}$ Max-Planck-Institute for Astrophysics, Karl-Schwarzschild-Str. 1, 85741 Garching, Germany \\ email: fk@mpa-garching.mpg.de
}

\begin{abstract}
A summary of the round table discussion following Session A on Modelling Convection and Radiative Transfer is provided based on the video recording made during the conference.
\end{abstract}

The panel for the round table discussion of Session A on Modelling Convection and Radiative Transfer was formed by the following speakers: V.M. Canuto, M. Steffen, J. Truillo Bueno, S. Wedemeyer-Böhm, and F. Rincon. It was chaired by the author of this summary, F. Kupka.

A number of questions were put forward by the chair person to stimulate discussion on issues raised during the talks given as part of Session A.

The first question related to the terms "eddies", "plumes", and "drafts". How important are these concepts? Are they just terminology and what physics implied by these terms which makes them different from each other in a fundamental way?

F. Rincon noticed that though some coherent structures can have special transport properties, it can be misleading to individualize features in a dynamical system such as a turbulent flow. Plumes and eddies are not necessarily paradoxial concepts, since features such as coherent downdrafts are observed in convective flows studied by different scientific communities, and the regions of highest vertical vorticity are located within those plumes which characterise strong downflows.

V. Canuto agreed and mentioned the question of coherent structures vs. one-point closure modelling was already discussed by Lumley, who said that one has to get out of the conceptual predicament of stopping at second order moments in one-point closure models. $\dagger$ As long as one stops at second order, there can't be anything left of coherent structures in the model, but — as V. Canuto remarked — that's like stopping a Fourier expansion at the first term. This is not an indictment against the methodology, but just caused by stopping the methodology at too low order. The higher order moments bring in the dynamics, which is visible as plumes. So 'this is not a question of methodology, but a question of how far you go. It is true that for many years we did not know how to deal with these higher order moments, because a) we did not have experimental data and b) because we did not know how to deal with them. The situation has dramatically changed in the last few years, because we have LES data and laboratory data of higher order moments, and therefore we can "plumenize" the equations, if I may repeat my terrible verb. If you want to read the equations in the language of plumes that's fine.' Coming back to the question of vorticity created by the small scales he pointed out they create a lot of vorticity, a lot of dynamics, are advective, when moving downwards, which is completely outside of the Kolmogorov range, but that per se does not limit the one-point closure models.

$\dagger$ such as mixing length theory 
H.-G. Ludwig pointed out that plumes are geometrical structures and wondered how they could be 'seen' in the turbulence models. V. Canuto responded to this question by mentioning that one has to make a choice in turbulence modelling. One can "plumenize" the model at different orders and he chose to do so at the third order. Once this is done, a simplification is introduced, namely, an assumption about the probability distribution function, but one avoids having to use even higher orders. At that point also a geometrical factor comes in: what is the space covered by the plume? For that a dynamical equation is needed and that is part of the model, since the system is already closed at that point. This is the advantage of using a turbulence model compared to the phenomenological approach used in earlier plume models. The old models such as those introduced by Taylor also could not deal with the amount of entrainment other than through an adjustable parameter, but starting from the moment equations the entrainment fortunately is automatically part of the dynamical equations of the model. H.-G. Ludwig then asked how in the model the vertical extension of the plume is determined. V. Canuto responded that this basically comes out of the solution of the equations, specifying the point where the plume is 'dead' as the point where the size of the plume goes to $1 / 2$. At that point the plume is indistinguishable from its environment. This geometrical factor comes out as a solution of the equations rather than being prescribed externally.

Time constraints implied the discussion had to move on to the next topic. Since global simulations where to be discussed in more detail later during the week, questions concerning that topic were left for the subsequent round tables.

The second question posed by F. Kupka to the panel and the audience related to the fact that in most of the hydrodynamical simulations of surface convection the magnetic field is being neglected and nevertheless the simulations are being used for extremely high precision abundance determinations. With this background as a basis the question posed was: in which cases are we on the save side, if we neglect magnetic fields, both in the simulations themselves and in the abundance analyses done on their basis?

M. Steffen pointed out that most of the area of the quiet Sun has field strengths much less than required to form features such as flux tubes, i.e., the so-called plasma- $\beta$ is much larger than 1, so the flow itself won't be affected. So in this sense we are on the save side for abundance analysis. That is different for the chromosphere, of course. F. Kupka then wanted to double-check: are there nevertheless lines which should be avoided in abundance analyses because of the solar mean magnetic field? J. Truillo Bueno replied that abundance analyses in 3D models are biased towards the upflow regions, where the field is very weak. So he would not expect in principle a big difference between results from simulations with and without a magnetic field. Martinez Pillet then posed a question to J. Truillo Bueno: if there is a magnetic field of $100 \mathrm{G}$ in the magnetically quiet region filling a resolution element of the detector, it will cause a Zeeman broadening of Stokes $I$. This won't create much polarization, but it will create Zeeman broadening — so should not that influence abundance determinations of 0.04 dex accuracy or better? Quoting earlier results by Stenflo and Lindegren J. Truillo Bueno pointed out that lines in the visible are not sensitive enough, though some in the infrared are and promised results on that to be published soon. This, too, indicates that current radiation hydrodynamics based abundance analyses are on the save side. J.-P. Zahn then asked whether even such a weak field should not create a magnetic pressure and thus change stratification? This question did not receive a specific answer, but J. Truillo Bueno mentioned that he expects field strengths found to be $20 \%$ weaker than in his current analysis, if the latter is done in a consistent way with $3 \mathrm{D}$ radiation magneto-convection simulations.

The third question posed by F. Kupka related to the expected necessity of doing radiative transfer calculations in a non-LTE framework for numerical simulations that 
include the chromosphere, since for the latter the local thermal equilibrium assumption breaks down completely. 'Have comparisons between LTE and non-LTE calculations been done for this case, of 3D simulations and also with 1D ones?'

$\mathrm{S}$. Wedemeyer-Böhm replied that of course non-LTE radiative transfer is required for the chromosphere. That has been computationally too expensive, though in a next step at least certain lines and atomic species should be treated in such detail. Comparisons with $1 \mathrm{D}$ models would be of interest, if they can be done for the 3D case with the same setting. Right now, with the present settings, properities like the temperature fluctuations are a bit uncertain. Ca resonance lines would provide a particularly meaningful diagnostics, since they are formed over a larger depth range, but at the moment this is out of reach.

The question was moved on to M. Steffen in relation to results shown about metal poor stars. He pointed out that the Li I line in the red used for abundance determinations is known to be strongly affected by non-LTE effects, so abundance corrections with 3D simulations based on LTE calculations may be misleading, but other lines such as molecular lines, are much less affected and in some cases they lead to large corrections of 1D based calculations.

F. Kupka then mentioned the poster on RHD simulations of A-stars by Kochukhov et al. displayed in the poster presentation room. Could part of the problems mentioned there be due to non-LTE effects, or are they rather due to non-grey radiative transfer effects or other problems in the temperature structure? The question was taken up by O. Kochukhov who pointed out that the structure of the 3D model is very similar to the $1 \mathrm{D}$ case, and since the differences between LTE and non-LTE are small in that case, he'd expect non-LTE effects to be not very important. M. Steffen added that still it might be interesting to check that, since in 3D effects could be larger due to temperature differences between up- and downflows, although he does not believe that this is really important in that case. N. Piskunov pointed out that non-grey calculations increase significantly the horizontal velocities and while this helps to fit weak lines, strong lines still cannot be matched. This opens the possibility that non-LTE could play a role for the strong lines right on the stellar surface.

The fourth and last major question put by F. Kupka to the panel concerned their opinion on the usefulness of numerical simulations in calibrating free parameters used in $1 \mathrm{D}$ models such as micro- and macroturbulence velocities and mixing length $\alpha$. Would it be feasible to do that at least for the giant and the dwarf stars over the entire HR diagram, from A to M type?

M. Steffen stated that in principle one can calibrate $\alpha$, but only for stellar evolution calculations, which is not the same as for the stellar atmospheric structure. One can do that for stars with deep reaching convection zones. Microturbulence 'comes out of the simulations', so one could make a grid for that. F. Kupka then asked what would be the main bottleneck in such an effort: computing power, the number of people running the codes or doing the comparisons, or all of them together? Model grids would require thousands of models accounting for different metallicities and the like. M. Steffen expects that all of them would matter, although some regions of the HR diagram (solar-like stars) are easier than other (low gravity stars and hotter stars). I. Roxburgh pointed out he does not like the phrase "calibrate the mixing length", since "what you really mean is calibrate the entropy on an adiabat.' Otherwise, 'some unjustified credence is given to the use of the word mixing length.' He also mentioned that such a grid has partially been done by Nordlund and Stein. R. Stein pointed out that several others have been involved in that work including $\mathrm{R}$. Trampedach. Other work in that direction was done by H.-G. Ludwig and collaborators. H.-G. Ludwig responded that for calibrating the mixing length mostly the thermal structure matters. The latter one gets out of the 3D 
calculations rather easily, as resolutions of only 100 or 200 grid points in the horizontal directions are sufficient to constrain that. He also concluded that the limitation is hence rather in the number of people working on the problem. V. Canuto disagreed on the whole effort and called it a hopeless exercise: 'Frankly, the mixing length belongs to the past. We don't use horse-carriages any more, we use the subway.' H.-G. Ludwig replied that the calibration of the mixing length should be interpreted in the sense just discussed with I. Roxburgh before and there may still be some use in it for differential comparisons with $1 \mathrm{D}$ models, also for practical reasons, since it is widely spread. V. Canuto 'just expected to disagree with him on that.' Which ended the debate, since time for discussion was over.

In conclusion, despite there is some convergence in viewpoints on what is physically most important to model a stellar convective flow, despite all the progress in the field of simulations and new models for turbulent convection becoming available over the recent years which incorporate more physics than the classical models, the issue of how to best carry over this progress to stellar modelling at large is still a point which sparks strong disagreements. More debates along this line took place later on during the symposium and the round table discussion sessions which followed this opening one. 